\title{
Information Management and Applications of Intelligent Transportation System
}

\author{
Chi-Chun Lo, ${ }^{1}$ Kuo-Ming Chao, ${ }^{2}$ Hsu-Yang Kung, ${ }^{3}$ Chi-Hua Chen, ${ }^{1,4,5}$ and Maiga Chang ${ }^{6}$ \\ ${ }^{1}$ Department of Information Management and Finance, National Chiao Tung University, 1001 University Road, Hsinchu 300, Taiwan \\ ${ }^{2}$ Department of Computing, Coventry University, Priory Street, Coventry CV1 5FB, UK \\ ${ }^{3}$ Department of Management Information Systems, National Pingtung University of Science and Technology, \\ 1 Shuefu Road, Neipu, Pingtung 912, Taiwan \\ ${ }^{4}$ Telecommunication Laboratories, Chunghwa Telecom Co., Ltd., 99 Dianyan Road, Yangmei District, Taoyuan 326, Taiwan \\ ${ }^{5}$ Department of Communication and Technology, National Chiao Tung University, 1001 University Road, Hsinchu 300, Taiwan \\ ${ }^{6}$ School of Computing and Information Systems, Athabasca University, 1 University Drive, Athabasca, AB, Canada T9S $3 A 3$
}

Correspondence should be addressed to Chi-Hua Chen; chihua0826@gmail.com

Received 5 August 2015; Accepted 11 August 2015

Copyright (C) 2015 Chi-Chun Lo et al. This is an open access article distributed under the Creative Commons Attribution License, which permits unrestricted use, distribution, and reproduction in any medium, provided the original work is properly cited.

\section{Introduction}

The rise of economic growth and technology advance has led to increasing demand of the intelligent transportation system (ITS) for traffic service. How to construct real-time information systems of ITS has become more important [1]. Real-time traffic information, such as average vehicle speed, travel time, traffic flow, and traffic congestion, can be used by road users and the ministry of transportation to improve the level of service for road ways. Several approaches have been developed to collect and send realtime traffic information to traffic information centre via various networks (e.g., vehicular ad hoc network (VANET) [2], universal mobile telecommunications system (UMTS) [3], and long-term evolution (LTE) [4]): vehicle detector [5], global position system- (GPS-) based probe car reporting [6], cellular floating vehicle data (CFVD) [7], and so forth. Furthermore, information and communications technology (ICT) can be used to analyse the real-time traffic information to forecast the future traffic condition for road user decision. Therefore, the aim of this special issue is to introduce for the readers a number of papers on various aspects of traffic information management.

Topics covered in this issue include three main parts: (1) traffic information estimation and prediction, (2) transportation safety and security, and (3) logistics transportation traffic management. This special issue has received a total of 32 submitted papers with only 5 papers accepted. A high rejection rate of $84.38 \%$ of this issue from the review process is to ensure that high-quality papers with significant results are selected and published. The three topics and accepted papers are briefly described below.

\section{Traffic Information Estimation and Prediction}

Papers on analytical methods for traffic information estimation and prediction are as follows: (1) "A Method for Driving Route Predictions Based on Hidden Markov Model” by N. Ye et al. and (2) "Detecting Traffic Anomalies in Urban Areas Using Taxi GPS Data” by W. Kuang et al.

N. Ye et al. from China and South Africa in "A Method for Driving Route Predictions Based on Hidden Markov Model” proposed a driving route prediction method based on hidden Markov model (HMM) to predict the traffic condition of each road segment for driver's reference. Furthermore, a method of training set extension based on $K$-means++ and a smoothing technique was used to build the HMM for route predictions. In their experimental environment, several training and test examples in Jiangsu, China, were selected to evaluate their proposed method. The experimental results illustrated that 
the correct prediction rate of their proposed method could be high.

W. Kuang et al. from China in "Detecting Traffic Anomalies in Urban Areas Using Taxi GPS Data" proposed a traffic anomalies detection method which could combine the wavelet transform method and principal component analysis (PCA) to detect traffic anomalies. Moreover, their proposed method could estimate and obtain information regarding the spatial distribution of traffic flows. In their experimental environment, several taxicabs collected and reported their GPS data in Harbin, China, for the evaluation of their proposed method. The experimental results indicated that a number of the traffic anomalies could be detected and reported for managers to solve traffic jam.

\section{Transportation Safety and Security}

Paper on analytical methods for transportation safety and security is presented as follows. S.-K. Ryu et al. from Korea in "Image-Based Pothole Detection System for ITS Service and Road Management System" proposed a pothole detection method based on various features in two-dimensional (2D) images which included three steps: (1) segmentation based on Histogram Shape-Based Thresholding (HST), (2) candidate region extraction in accordance with various features (e.g., size and compactness), and (3) decision by comparing pothole and background features. In their experimental environment, several video clips in Korea were selected to evaluate their proposed method. The experimental results showed that the accuracy, precision, and recall of their proposed method were higher than previous methods.

\section{Logistics Transportation Traffic Management}

Papers on analytical methods for logistics transportation traffic management are as follows: (1) "Identifying Key Factors for Introducing GPS-Based Fleet Management Systems to the Logistics Industry" by Y.-C. Hu et al. and (2) "Novel Encoding and Routing Balance Insertion Based Particle Swarm Optimization with Application to Optimal CVRP Depot Location Determination" by R.-M. Chen and Y.-M. Shen.

Y.-C. Hu et al. from Taiwan in "Identifying Key Factors for Introducing GPS-Based Fleet Management Systems to the Logistics Industry" combined decision-making trial and evaluation laboratory (DEMATEL) and analytic network process (ANP) to determine the key indicators (e.g., funding and budget, experience and ability of consultants, project team composition, user recognition, timely and correct information, and degree of completeness of transmission equipment) for introducing GPS-based fleet management systems to transport companies. In their experimental environment, a transport company in Taiwan was selected to evaluate their proposed method. The experimental results indicated that adequate annual budget planning, enhancement of user intention, and collaboration with consultants were the key indicators for successfully introducing the systems.
R.-M. Chen and Y.-M. Shen from Taiwan in "Novel Encoding and Routing Balance Insertion Based Particle Swarm Optimization with Application to Optimal CVRP Depot Location Determination" proposed a hierarchical particle swarm optimization (PSO) with two layers (i.e., outer layer PSO and inner layer PSO) for the establishment of the optimal depot location and the minimized total distance of vehicle routing. In their experimental environment, nine instances were selected from an accessible and credible database which was designed by Augerat for the evaluation of vehicle routing algorithm. The experimental results illustrated that the costs of finding the new plant location and vehicle routing distance in a real world case could be reduced.

Chi-Chun Lo Kuo-Ming Chao Hsu-Yang Kung Chi-Hua Chen Maiga Chang

\section{References}

[1] K. Boriboonsomsin, M. J. Barth, W. Zhu, and A. Vu, "Ecorouting navigation system based on multisource historical and real-time traffic information," IEEE Transactions on Intelligent Transportation Systems, vol. 13, no. 4, pp. 1694-1704, 2012.

[2] X. Ma, J. Zhang, X. Yin, and K. S. Trivedi, "Design and analysis of a robust broadcast scheme for VANET safety-related services," IEEE Transactions on Vehicular Technology, vol. 61, no. 1, pp. 46-61, 2012.

[3] A. Bazzi, B. M. Masini, and O. Andrisano, "On the frequent acquisition of small data through RACH in UMTS for its applications," IEEE Transactions on Vehicular Technology, vol. 60, no. 7, pp. 2914-2926, 2011.

[4] K. Zheng, F. Liu, Q. Zheng, W. Xiang, and W. Wang, "A graphbased cooperative scheduling scheme for vehicular networks," IEEE Transactions on Vehicular Technology, vol. 62, no. 4, pp. 1450-1458, 2013.

[5] B.-F. Wu and J.-H. Juang, "Adaptive vehicle detector approach for complex environments," IEEE Transactions on Intelligent Transportation Systems, vol. 13, no. 2, pp. 817-827, 2012.

[6] B. Tian, B. T. Morris, M. Tang et al., "Hierarchical and networked vehicle surveillance in ITS: a survey," IEEE Intelligent Transportation Systems Magazine, vol. 16, no. 2, pp. 557-580, 2015.

[7] M.-F. Chang, C.-H. Chen, Y.-B. Lin, and C.-Y. Chia, "The frequency of CFVD speed report for highway traffic," Wireless Communications and Mobile Computing, vol. 15, no. 5, pp. 879888, 2015. 


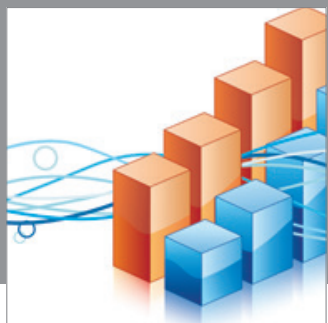

Advances in

Operations Research

mansans

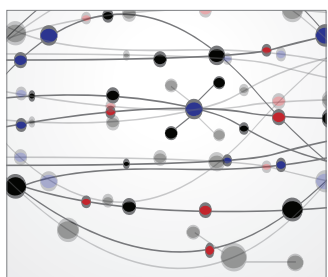

The Scientific World Journal
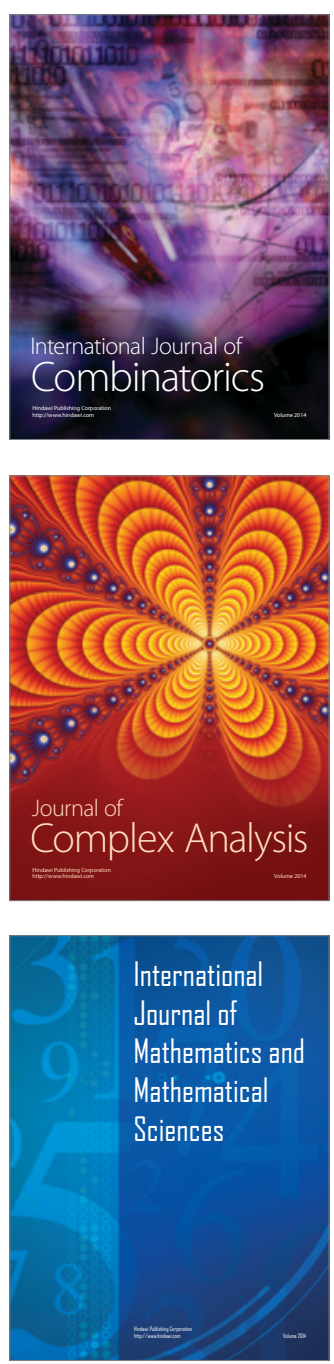
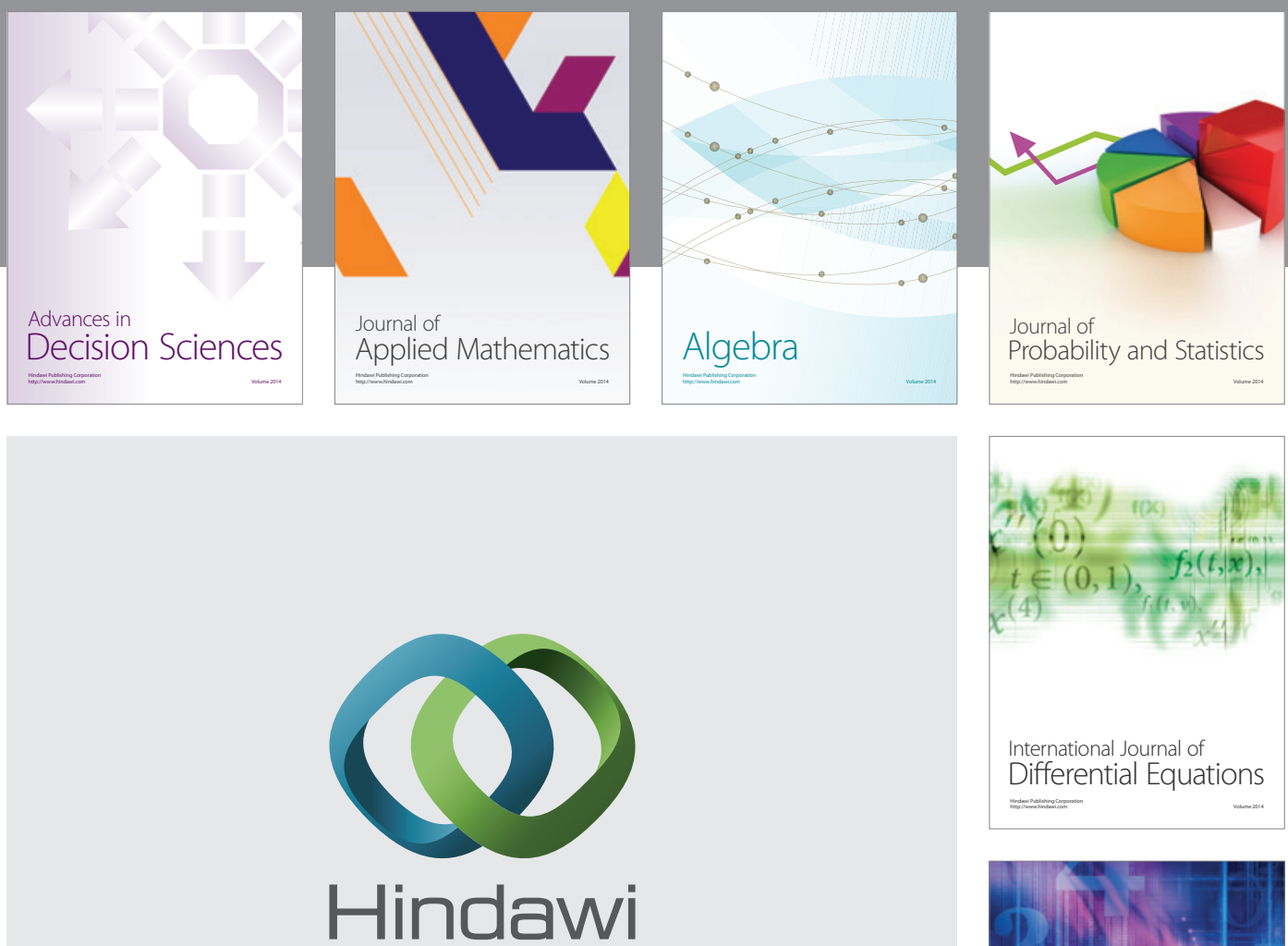

Submit your manuscripts at http://www.hindawi.com
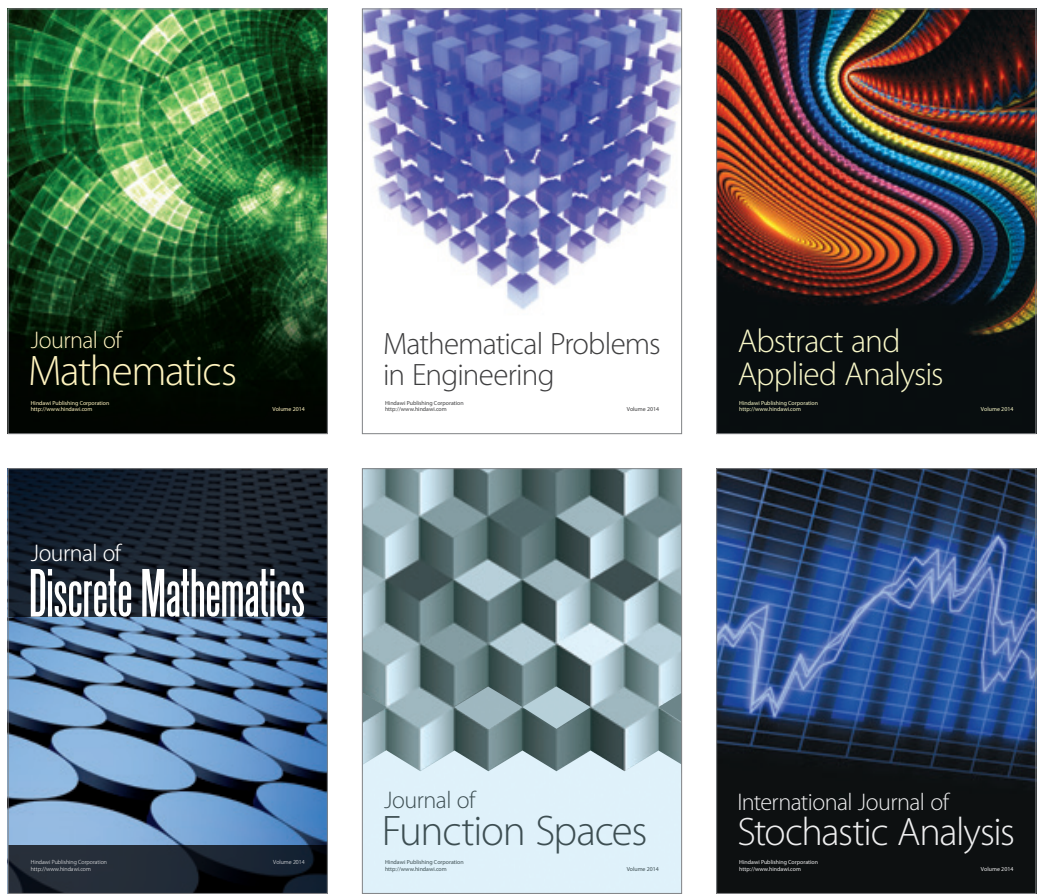

Journal of

Function Spaces

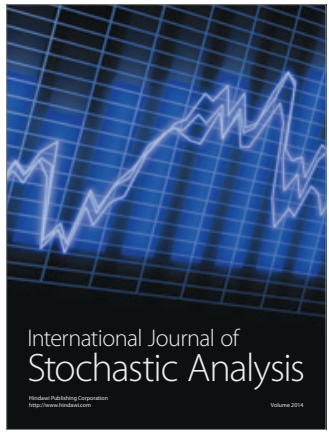

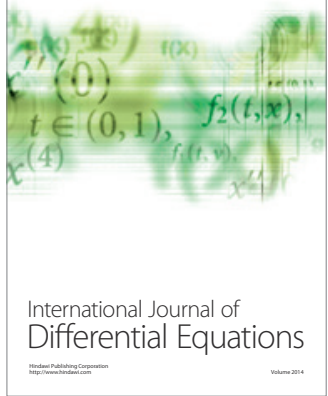
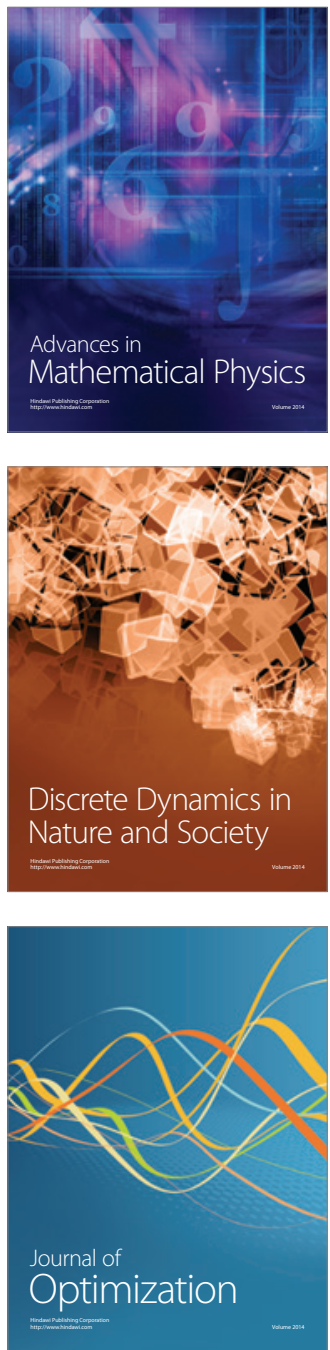